\title{
Serum REG3alpha and C-Reactive Protein Levels in Crohn's Disease Patients Undergoing Immunoablation and Autologous Hemopoetic Stem Cell Transplantation in the ASTIC Trial
}

\author{
Thomas Greuter $^{\mathrm{a}}$ Silvia Lang ${ }^{\mathrm{a}}$ Ernst Holler ${ }^{\mathrm{b}}$ Christopher J. Hawkey ${ }^{\mathrm{c}}$ \\ Gerhard Rogler $^{\text {a }}$ Luc Biedermann $^{\text {a }}$ ASTIC trial group \\ a Division of Gastroenterology and Hepatology, University Hospital Zurich, Zurich, Switzerland; ${ }^{\mathrm{b}}$ Department of \\ Hematology/Oncology, University Medical Center Regensburg, Regensburg, Germany; ${ }^{C}$ Nottingham Digestive \\ Diseases Centre, University of Nottingham, Nottingham, UK
}

\section{Key Words}

Autologous hemopoetic stem cell transplantation · Crohn's disease - Serum biomarker - Graft-versus-host disease .

Regenerating islet-derived 3-alpha

\begin{abstract}
Background: REG3a has been recently shown to be a highly accurate biomarker for graft-versus-host-disease (GvHD). Given the unmet need of such biomarkers in Crohn's disease (CD) and the similarities between CD and GvHD, we aimed at investigating the role of serum REG3a as a diagnostic and prognostic biomarker in $\mathrm{CD}$ patients undergoing autologous hemopoetic stem cell transplantation (HSCT) in the multicenter Autologous Stem Cell Transplantation International Crohn's Disease (ASTIC) trial and to compare it to C-reactive protein (CRP). Methods: Stored serum samples from the ASTIC trial were analyzed using a commercially available human PAP1 ELISA-kit to measure REG3a levels. CRP was available from prior analysis in the ASTIC trial. These levels were correlated with clinical and endoscopic disease activity as well as overall clinical and endoscopic outcome 1 year after autologous HSCT. Results: One hundred thirty two serum samples were available for analysis. The mean concentration of REG3a was $101.8 \mathrm{ng} / \mathrm{ml}(95 \% \mathrm{Cl}$
\end{abstract}

22.6-258.3). No significant elevation of REG3a was found among patients with active disease compared to those in remission (106.3 vs. 91.4). Patients with moderate to severe endoscopic disease activity showed substantially, although not significantly elevated REG3a levels compared to those in remission (95.4 vs. 52.4, $p=0.052$ ). Baseline serum REG3a levels of patients without clinical or endoscopic remission 1 year after HSCT were not elevated compared to those in remission (63.1 vs. 66.9 , and 68.4 vs. 59.2 , respectively). In contrast, CRP was significantly elevated in patients with active disease compared to patients in remission (14.1 vs. $6.0 \mathrm{mg} / \mathrm{dl}, \mathrm{p}=0.032$ ). In addition, CRP was elevated, although not significantly, in patients with severe endoscopic disease compared to those in endoscopic remission (18.7 vs. 4.1, $p=0.062$ ). Furthermore, baseline CRP was reduced in patients with clinical and endoscopic remission after HSCT compared to those without remission, although not significantly ( 8.8 vs. 21.4 , n.s. and 8.1 vs. 12.4 , n.s.). Conclusion: Given the divergent findings compared to GvHD, we conclude that serum REG3a is not an accurate diagnostic and predictive biomarker in CD patients undergoing HSCT. In contrast, CRP is a valuable biomarker in order to differentiate active disease from remission. However, CRP does not seem to be of prognostic value for HSCT outcome.

(c) 2015 S. Karger AG, Basel

\section{KARGER 125}

(c) 2015 S. Karger AG, Base

0012-2823/15/0922-0083\$39.50/0 


\section{Introduction}

The 3 REG3 genes alpha, beta, and gamma all seem to be involved in the growth of cells and tissues in the gastrointestinal tract [1]. The human equivalent of REG3a is HIP/PAP [2, 3] and is located on chromosome 2 [1]. It encodes C-type lectine-like proteins, which are secreted into the gut lumen by Paneth cells $[4,5]$. Due to their antimicrobial activity, REG3a protein seems to support the epithelial barrier function in the gastrointestinal tract [5]. Interestingly, gastrointestinal inflammation leads to an increased secretion of REG3a via increased interleukin-22 (IL-22) [6]. However, commensal microflora is required for the increase in IL-22 production [7]. Hence, IL-22 has been shown to be low in germ-free mice. IL-22 stimulates the production of S100a and REG3 $\alpha$ (both involved in antimicrobial defense of epithelial cells) via its IL-22 receptor [6]. Given the fact that the IL-22 receptor is exclusively expressed on intestinal epithelial cells, it is considered to be an important factor in the communication between immune and intestinal epithelial cells [8]. Moreover, the so-called IL-22/REG3-axis has been shown to be the first known molecular pathway, enlightening how immune cells instruct and communicate with epithelial cells [7].

Graft-versus-host-disease (GvHD) shares many similarities (histological features and genetic risk factors) with Crohn's disease (CD) [9]. Mucosal damage in GvHD increases REG3a, which can transverse consecutively into systemic circulation. Recently, REG3 $\alpha$ has been shown to be a highly accurate diagnostic and prognostic biomarker with remarkable specificity for lower gastrointestinal GvHD after allogeneic hemopoetic stem cell transplantation (HSCT), allowing better risk stratification of patients and the prediction of non-relapse mortality [10]. Serum REG3 $\alpha$ seems to be a surrogate marker for the cumulative area of damage. Hence, it seems to be a direct indicator of intestinal mucosal damage rather than just activated systemic inflammatory response like C-reactive protein (CRP) or fecal calprotectin [10]. In a previous investigation in patients undergoing allogeneic HSCT, the transplant-related mortality revealed to increase stepwise in donor/recipient pairs with NOD2/CARD15 mutations either only in the donor or only in the recipient or in both compared to pairs without mutated single nuclear polymorphisms $[11,12]$. This is of particular interest given the fact, that NOD2 variant patients have significantly increased REG3a levels compared to NOD2 wild type patients. Hence, REG3a may also have a prognostic value in allogeneic HSCT without the complication of GvHD.
While knowledge about the role of REG3a in CD pathogenesis is relatively new, CRP has been studied for years. CRP has been shown to be associated with disease activity in CD and increased CRP levels are associated with higher response rates to anti-TNF treatments [13]. However, CRP can be elevated in virtually all inflammatory disorders and therefore, it is very unspecific for $\mathrm{CD}$ alone. In addition, in some individuals, CRP may not be elevated due to genetic variants $[14,15]$. And - in contrast to REG3a - specificity and sensitivity for GvHD have been shown to be very low [16]. Despite these limitations, CRP remains the best, but imperfect serum biomarker for disease activity in $\mathrm{CD}$. However, accurate non-invasive biomarkers for $\mathrm{CD}$ activity and mucosal damage are still an unmet need. Particularly, this seems to be true in the context of recent findings, suggesting a low correlation between symptom severity and endoscopic disease activity [17].

Autologous HSCT has been shown to be an effective treatment option in a variety of severe autoimmune diseases refractory to conventional therapy [18]. The Autologous Stem Cell Transplantation International Crohn's Disease (ASTIC) trial is the only randomized controlled trial investigating the role of autologous HSCT in CD. The trial was conducted as a multicenter study in 10 different countries and revealed a substantial reduction in symptomatic and endoscopic activities in severe CD after autologous HSCT compared to mobilization alone [1921]. In this study, we aimed at investigating the role of the novel promising biomarker REG3 $\alpha$ in order to assess its diagnostic and prognostic potential in CD patients undergoing immunoablation and autologous HSCT in the multicenter ASTIC trial and to compare this role to CRP, the so far best, but imperfect CD serum marker [13].

\section{Methods}

Patients and Samples

During the ASTIC trial, heparinized serum samples were collected prospectively and stored at the University of Nottingham at $-80^{\circ} \mathrm{C}$. Sample processing and storage were performed as previously described [22]. Samples were shipped from Nottingham to Zurich and were received frozen on dry ice. No sample was thawed more than once before analysis. Serum samples were analyzed using a commercially available human PAP1 ELISA-kit to measure serum REG3a levels. Clinical, laboratory (including CRP), and endoscopic data were available from the ASTIC trial.

ASTIC Trial

The ASTIC trial, a randomized controlled, multicenter study in 10 different countries, was conducted from July 2007 to May 2013 as to investigate the role of autologous HSCT in severe CD. The 
study was approved by the EBMT/EULAR Working Party Autoimmune Diseases, ECCO Scientific Committee and by the relevant Competent Authorities, Ethical Committees and Institutional Review Boards. Forty-five patients were enrolled. Inclusion criteria were (1) age between 18 and 50 years, (2) confirmed diagnosis of $\mathrm{CD}$ based on typical radiological appearances and/or typical histology, (3) active disease at registration defined as CDAI $>250$ and $>2$ of the following: raised CRP, endoscopic evidence of active disease confirmed on histology, clear evidence of small bowel CD on small bowel barium study, (4) unsatisfactory course despite 3 immunosuppressive agents, (5) impaired function and quality of life, (6) current problems unsuitable for surgery, (7) informed consent. Patients were excluded for (1) pregnancy, (2) concomitant severe disease such as renal failure, congestive heart failure, psychiatric disorders, (3) diarrhea due to short small or large bowel, (4) infection or risk thereof, (5) significant malnutrition, (6) previous poor compliance. All patients received mobilization treatment (intravenous cyclophosphamide over 2 days, then filgrastim daily) prior to randomization into either immediate (1 month) or delayed (13 months) immunoablation and autologous HSCT. All patients were followed up by 6-weekly assessments for 52 weeks after transplantation. At every follow-up visit, clinical disease activity was measured by CDAI [23] and HBI [24]. At baseline, endoscopic disease activity was assessed by upper endoscopy and ileocolonoscopy using CDEIS [25]. Further assessment tools consisted of adverse events evaluation, quality-of-life questionnaires, evaluation of medication use, and laboratory parameters (CRP, platelet count).

ELISAs

REG3a ELISA kits from MBL International Corporation (Ab-Match Assembly Human PAP1 kit, catatolog number 5323, and Ab-Match Universal kit, catalog number 5310, Woburn, Mass., USA) were used. All measurements were performed according to the manufacturer's protocol. All samples were diluted 1:10 according to the protocol and as recommended, all calibrators, controls, and patient samples were analyzed in duplicate. One standard curve per assay was made. Absorbance of each well was read at test wavelength of 450 and $620 \mathrm{~nm}$ with the multi detection microplate reader Synergy 2 (Biotek Instruments, Winooski, Vt., USA). Results were calculated with Gen 5 version 1.11 (Biotek Instruments). Only values within the range of the standard curve were used for further analysis. If duplicates varied more than $16 \%$, ELISA was repeated for this sample.

\section{Statistical Analysis}

Serum REG3 $a$ and CRP levels at any timepoint during the ASTIC trial were correlated with clinical (CDAI) and endoscopic disease activity (CDEIS). In addition, baseline serum REG3 $\alpha$ and CRP were correlated with the overall outcome 1 year after autologous HSCT in patients randomized to the immediate transplantation group (see section ASTIC trial), which was assessed by changes in CDAI and CDEIS, and by clinical (CDAI $\leq 150)$ or endoscopic remission (CDEIS <4), respectively, in order to evaluate its prognostic potential. For statistical analysis, the IBM Software SPSS Statistics version 22.0.0 (2013 SPSS Science, Inc., Chicago, Ill., USA) was used. The Mann-Whitney U test was used for comparison between different subgroups as data was ordinary or continuous with a non-normal distribution. To investigate the prognostic potential of REG3 $\alpha$ and CRP, baseline serum levels in patients with clinical and/or endoscopic remission were compared
Table 1. Baseline demographic data

\begin{tabular}{lcr}
\hline & $\mathrm{n}=34^{*}(95 \% \mathrm{CI})$ & \multicolumn{1}{c}{$\%$} \\
\hline Sex, female & 20 & 58.8 \\
Mean age, years & $32.0(20.9-45.7)$ & \\
Ethnicity: Caucasian & 34 & 100.0 \\
Allergy/atopic diseases & 18 & 52.9 \\
Positive family history for IBD & 5 & 14.7 \\
Current smoker & 7 & 20.6 \\
Past smoker & 12 & 35.3 \\
Ileostomy & 9 & 26.5 \\
Colectomy & 13 & 38.2 \\
Ileal resection & 10 & 29.4 \\
Operations & $2.4(0.0-6.0)$ & \\
Mean CDAI at baseline & $338.3(115.3-512.4)$ & \\
Mean CDEIS at baseline & $12.9(0.0-26.0)$ & \\
Mean CRP at baseline, mg/dl & $20.0(0.5-109.0)$ & \\
Mean REG3 $a$, ng/ml & $85.6(21.7-220.7)$ & \\
\hline
\end{tabular}

* Data of one patient was missing.

with serum levels in patients with no remission by using the Mann-Whitney U test. A 2 -sided p value of $<0.05$ was considered statistically significant.

\section{Results}

\section{Study Population}

As of January 2014, 132 serum samples were available from 35 of the 45 ASTIC trial participants. Twenty patients $(58.8 \%)$ were female and the mean age at mobilization was 32.0 years (95\% CI 20.9-45.7). All patients (100.0\%) had been previously treated with at least one TNF-blocker and $9(27.3 \%)$ were still treated with an anti-TNF at registration. Five patients (15.2\%) were on systemic steroids at study enrolment. By inclusion criteria, all patients had active disease at pre-registration. At baseline evaluation (within several weeks after registration) mean CDAI was 338.3 (95\% CI 115.3-512.4), only 3 patients reported to have a CDAI of $\leq 150$ at baseline. The mean endoscopic disease activity at baseline (CDEIS) was 1.9 (95\% CI 0.0-10.0) in upper endoscopy and 12.9 (95\% CI 0.0-26.0) in ileocolonoscopy. Baseline CRP was elevated with a mean of $20.0 \mathrm{mg} / \mathrm{dl}(95 \%$ CI 0.5-109.0). Demographic data is summarized in table 1.

\section{Serum REG3 $\alpha$ Levels at Baseline and during the ASTIC Trial}

The mean concentration of serum REG3 $\alpha$ at baseline was $85.6 \mathrm{ng} / \mathrm{ml}(\mathrm{n}=28,95 \%$ CI $21.7-220.7)$. The mean serum level of REG3 $\alpha$ at any timepoint was $101.8 \mathrm{ng} / \mathrm{ml}$ $(\mathrm{n}=132,95 \%$ CI 22.6-258.3). Changes in serum REG3 $\alpha$ 
Table 2. Mean REG3a levels and key clinical parameters according to low and elevated CDAI group

\begin{tabular}{lllll}
\hline $\begin{array}{l}\text { CDAI } \\
\text { group }\end{array}$ & $\begin{array}{l}\text { REG3a, mean, ng/ml } \\
(95 \% \mathrm{CI})\end{array}$ & $\begin{array}{l}\text { CDAI, mean } \\
(95 \% \mathrm{CI})\end{array}$ & $\begin{array}{l}\text { CRP, mean, } \\
\mathrm{mg} / \mathrm{dl}(95 \% \mathrm{CI})\end{array}$ & $\begin{array}{l}\text { CDEIS, mean } \\
(95 \% \mathrm{CI})\end{array}$ \\
\hline$\leq 150(\mathrm{n}=26)$ & $\begin{array}{l}91.4(16.7-243.1) \\
(\mathrm{n}=26)\end{array}$ & $\begin{array}{l}79.7(15.6-144.7) \\
(\mathrm{n}=26)\end{array}$ & $\begin{array}{l}6.0(0.1-21.0) \\
(\mathrm{n}=21)\end{array}$ & $\begin{array}{l}5.9(0.0-22.0) \\
(\mathrm{n}=11)\end{array}$ \\
$\geq 151(\mathrm{n}=74)$ & $\begin{array}{l}106.3(23.4-351.5) \\
(\mathrm{n}=74)\end{array}$ & $\begin{array}{l}297.2(162.3-507.6) \\
(\mathrm{n}=74)\end{array}$ & $\begin{array}{l}14.1(0.2-51.0) \\
(\mathrm{n}=49)\end{array}$ & $\begin{array}{l}10.7(0.0-26.0) \\
(\mathrm{n}=42)\end{array}$ \\
\hline $\mathrm{p}$ value & 0.289 & $<0.001$ & 0.032 & 0.075 \\
\hline
\end{tabular}

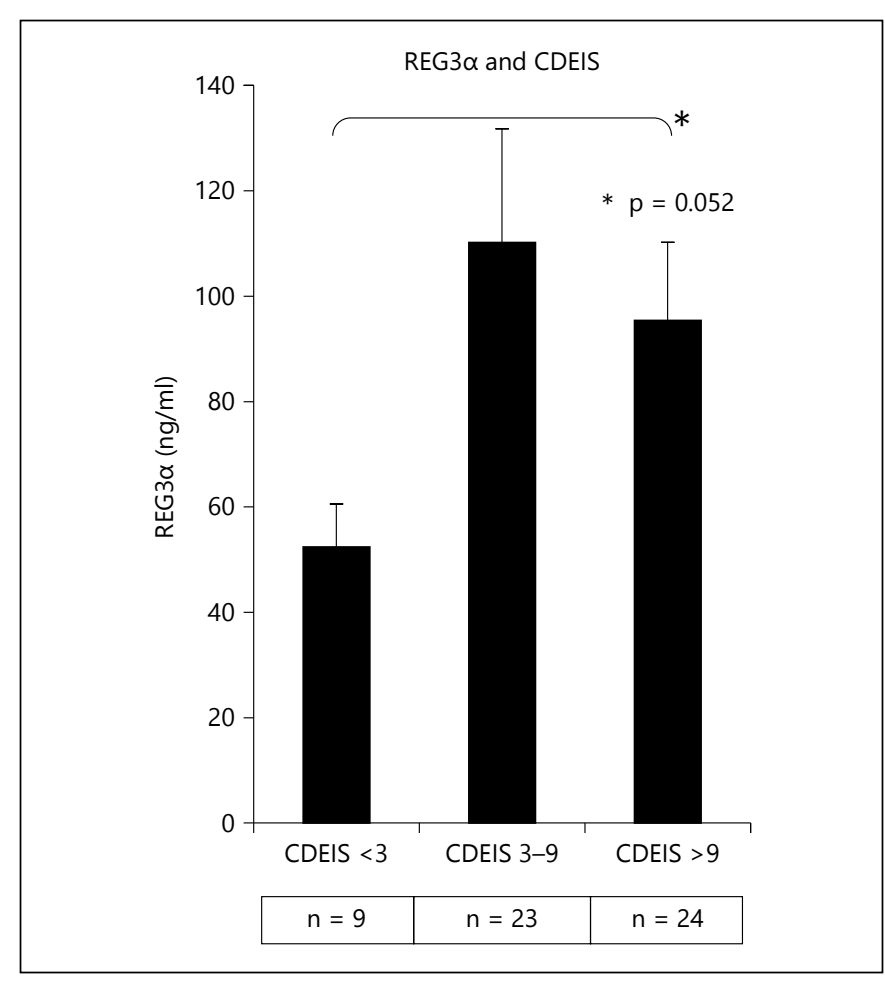

Fig. 1. Mean REG3a levels according to CDEIS group.

levels during the ASTIC trial are shown in online supplementary figure 1 (for all online suppl. material, see www. karger.com/doi/10.1159/000437300). Both baseline and overall serum REG3a levels were elevated compared to those of the normal population ( $\mathrm{n}=80$, reference $95 \% \mathrm{CI}$ $10.6-56.7 \mathrm{ng} / \mathrm{ml}$, according to manufacturer's protocol).

\section{Diagnostic Value of Serum REG3 $\alpha$ Levels in Severe \\ CD Patients}

CDAI at different timepoints during the ASTIC trial was correlated with serum REG3a levels. Therefore, we compared REG3 $\alpha$ levels of patients with a CDAI of $\leq 150$, indicating clinical remission with serum levels of patients with a CDAI of $\geq 151$, indicating active disease. A total of 26 CDAI of $\leq 150$ contrasted a number of 74 with a CDAI of $\geq 151$. No significant elevation of serum REG3 $a$ levels was found between these 2 groups (91.4 vs. 106.3). In contrast, CDEIS was relevantly elevated at timepoints with active disease compared to timepoints with clinical remission, although not significantly ( 10.7 vs. $5.9, \mathrm{p}=0.075$; table 2). Consecutively, endoscopic disease activity at different timepoints during the ASTIC trial was correlated with serum REG3 $\alpha$ concentration. REG3 $\alpha$ levels at timepoints with a CDEIS of more than 9, indicating moderate to severe endoscopic disease activity, were substantially although not significantly elevated compared to timepoints in endoscopic remission, defined as a CDEIS of less than 3 (95.4 vs. 52.4, $\mathrm{p}=0.052$ ). Likewise, serum from timepoints with mild endoscopic disease activity (CDEIS 3-9) did not show any significant elevation or reduction compared to endoscopic remission or severe endoscopic activity (110.2 vs. 52.4 , and 110.2 vs. 95.4 , respectively; fig. 1 ).

\section{Prognostic Value of Serum REG3 $\alpha$ Levels in CD \\ Patients Undergoing Immunoablation and \\ Autologous HSCT}

To evaluate the prognostic value of serum REG3 $\alpha$ concentration in the ASTIC trial, baseline levels of patients without clinical (CDAI $\geq 151, \mathrm{n}=8$ ) or endoscopic remission (CDEIS $\geq 4, n=5) 1$ year after autologous HSCT were compared with levels of patients in remission (clinical remission $n=6$; endoscopic remission, $n=6$ ). REG3 $a$ was analyzed only in samples from patients randomized to the early intervention group. Analysis did not show elevated serum levels in patients without future remission compared to patients with future remission after autologous HSCT (clinical remission 63.1 vs. 66.9, and endoscopic remission 68.4 vs. 59.2, respectively). Changes in CDAI or CDEIS after autologous HSCT did not show any correlation with REG3a levels at baseline assessment (fig. 2). 
Table 3. Diagnostic and prognostic value of CRP

\begin{tabular}{lcl}
\hline CRP values & Mean, mg/dl $(95 \%$ CI $)$ & p values \\
\hline Baseline CRP $(\mathrm{n}=23)$ & $20.0(0.5-109.0)$ & \\
CRP at any timepoint $(\mathrm{n}=70)$ & $11.6(0.1-46.0)$ & \\
Diagnostic value & $6.0(0.1-21.0)$ & 0.032 \\
$\quad$ CDAI $\leq 150(\mathrm{n}=21)$ & $14.1(0.2-51.0)$ & $0.376($ CDEIS $<3$ vs. CDEIS 3-9) \\
CDAI $\geq 151(\mathrm{n}=49)$ & $4.1(0.0-18.0)$ & $0.267($ CDEIS 3-9 vs. CDEIS >9) \\
CDEIS $<3(\mathrm{n}=7)$ & $8.6(0.0-44.0)$ & $0.062($ CDEIS $<3$ vs. CDEIS >9) \\
CDEIS 3-9 $(\mathrm{n}=16)$ & $18.7(0.7-58.0)$ & \\
CDEIS $>9(\mathrm{n}=21)$ & $8.8(0.0-22.0)$ & 0.689 \\
Prognostic value & $21.4(0.6-125.0)$ & 1.000 \\
Clinical remission $(\mathrm{n}=6)$ & $8.1(0.0-25.0)$ & \\
No clinical remission $(\mathrm{n}=9)$ & $12.4(0.9-41.0)$ & \\
Endoscopic remission $(\mathrm{n}=10)$ & & \\
No endoscopic remission $(\mathrm{n}=5)$ & & \\
\hline
\end{tabular}

Diagnostic and Prognostic Value of CRP in CD

Patients Undergoing Immunoablation and Autologous HSCT

Baseline CRP and CRP values throughout the ASTIC trial are shown in table 3 and in online supplementary figure 2, respectively. Both values were found to be elevated. In contrast to REG3 $\alpha$, CRP was elevated in patients with active disease (CDAI $\geq 151$ ) compared to those in clinical remission (CDAI $\leq 150)$. Difference was statistically significant ( 14.1 vs. $6.0, p=0.032)$. In order to assess CRP's potential to differentiate severe endoscopic disease activity from milder forms, CRP levels of different CDEIS groups were compared, which are shown in table 3. Patients with a CDEIS of more than 9 showed a relevantly elevated CRP compared to those with a CDEIS of less than 3 (18.7 vs. 4.1). However, the difference was not statistically significant $(\mathrm{p}=$ 0.062). Contrary to REG3a, baseline CRP was markedly reduced in patients with clinical or endoscopic remission 1 year after HSCT compared to those without remission (8.8 vs. 21.4, and 8.1 vs. 12.4). However, these differences did not reach statistical significance (table 3).

\section{Discussion}

REG3 $a$ has been shown to be a highly accurate biomarker specific for lower GvHD, a disease state harboring many similarities with $\mathrm{CD}$, through an unbiased, in-depth tandem MS-based discovery approach that can quantify proteins at low concentrations $[9,10]$. A leading hypothesis is that the epithelial barrier function in

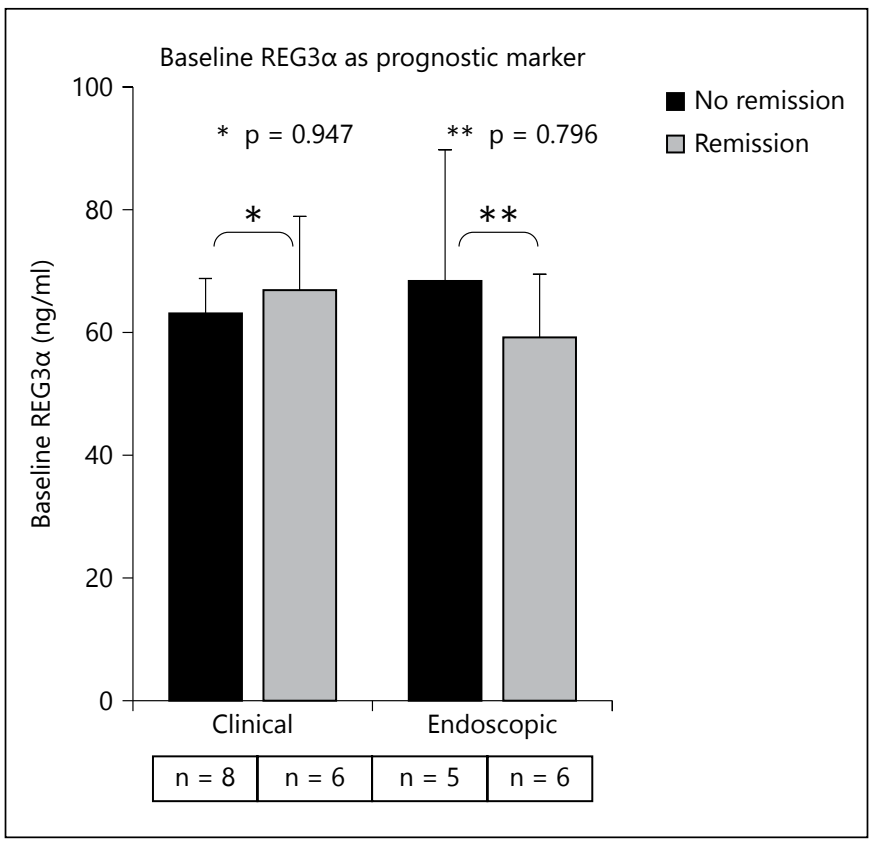

Fig. 2. Baseline REG3a levels in patients with and without clinical or endoscopic remission.

the gastrointestinal tract is supported by the antimicrobial activity of REG3 $\alpha$, which is secreted by Paneth cells into the crypt microenvironment [5]. In addition, secretion of REG3 $a$ is increased by gastrointestinal inflammation via IL-22 [6]. Mucosal damage could be correlated with high serum REG3a levels in GvHD, which suggests that breaches in the epithelial barrier caused by severe inflammation permit REG3 $a$ to traverse into the systemic circulation [10]. Therefore, REG3 $\alpha$ is thought 
to be a powerful indicator of the cumulative intestinal mucosal damage rather than just activated inflammatory response, a parameter impossible to measure by individual tissue biopsies [10]. Furthermore, baseline serum REG3a levels have been shown to be of high prognostic value regarding the outcome of GvHD therapy [10]. These features imply that REG3 $\alpha$ might serve as a valuable candidate biomarker in $\mathrm{CD}$. Given the fact, that accurate noninvasive biomarkers are still an unmet need in CD, this seems to be of clinical interest, in particular. However, in contrast to the shared similarities of GvHD with CD and the suggested surrogacy for intestinal mucosal damage - we did not observe any diagnostic or prognostic value of serum REG3a levels for severe CD in this study. Serum REG3a levels at timepoints with high CDAI scores indicating active disease were not elevated compared to timepoints with clinical remission. At least, we detected remarkably - although not significantly - higher serum REG3a levels in patients with severe endoscopic disease activity compared to those in endoscopic remission. Baseline serum REG3a levels of patients without clinical and/or endoscopic remission 1 year after autologous HSCT were not elevated compared to patients in remission indicating no prognostic utility of REG3a in the ASTIC trial. In contrast to REG3 $\alpha$, CRP distinctly discriminated between active disease and clinical remission based on symptom severity (assessed by CDAI). In addition, patients with severe endoscopic disease activity showed higher CRP levels than those in endoscopic remission, although this difference reached only borderline significance.

Our findings suggest that REG3 $a$ may be a more specific biomarker for GvHD and thus may be of no value for $\mathrm{CD}$. One possible explanation may be the different pathophysiology of GvHD compared to CD with intestinal stem cells (ISCs) as principal cellular targets and the consecutive lack of mucosal regeneration [26]. Paneth cells are in close proximity with ISCs, and this leads to high concentrations of REG3 $\alpha$ in this microenvironment in the presence of an inflammation. Therefore, mucosal barrier disruption induced specifically by stem cell dropout - as in GvHD - may allow REG3a to traverse into the systemic circulation [10]. So, different patterns of mucosal damage may lead to a different degree of REG3a secretion into the blood stream. Furthermore, highly active disease causing mucosal damage may also lead to a breakdown of Paneth cells, which may lower their potential to secrete REG3a into the gut lumen and consecutively into systemic circulation. In contrast, our data supports prior studies evaluating
CRP's role as the best, but imperfect serum marker for CD activity.

A limitation of this study certainly is the incomplete serum sample set with samples available from only 35 of the total 45 patients from the ASTIC trial. Also, serum samples of each individual patient were not stored from every timepoint throughout the ASTIC trial. This may have underpowered the analyses made in this study. Differences in CRP levels between patients with clinical/endoscopic remission after HSCT and those without remission might have reached statistical significance with a larger study population. The same might be true for the assessment of endoscopic disease activity by CRP and REG3a. Furthermore, our study included only selected patients with highly active disease, which is reflected by the high proportion of CDAI scores of more than 150 throughout the ASTIC trial. Based on the explanation of Paneth cell breakdown causing lower REG3a secretion given earlier, REG3a may have been more discriminative in patients with less active disease. In addition, NOD2 mutation status was not included in the analysis. NOD2 variant patients have been shown to have significantly higher REG3a levels as compared to NOD2 wild type patients. In addition, NOD2/CARD15 mutations are associated with higher mortality in patients undergoing allogeneic HSCT and have been shown to be an important prognostic factor $[11,12]$. Thus, subgroup analysis in patients with NOD2 variants would have been of special interest. REG3a might be more discriminative in these patients.

In conclusion, we did not observe a correlation between serum REG3a levels and CD activity except for a trend regarding endoscopic disease activity scores. In contrast to the findings in GvHD after allogeneic HSCT, baseline REG3 $\alpha$ did not have any prognostic value for clinical and endoscopic outcome 1 year after autologous HSCT. Thus, REG3a may be more specific for GvHD than previously thought. Given the divergent findings compared to GvHD, we conclude that REG3a is not a promising diagnostic and predictive biomarker in $\mathrm{CD}$ patients undergoing autologous autologous HSCT. However, further studies with higher case loads are needed to further investigate the diagnostic and prognostic role of serum REG3a in CD, also aside from autologous HSCT. In contrast to REG3 $\alpha, \mathrm{CRP}$ as the so far best serum marker in CD seems to be adequate for discrimination between active disease and clinical remission. However, CRP does not seem to be of prognostic value for HSCT outcome.
88

Digestion 2015;92:83-89 DOI: $10.1159 / 000437300$
Greuter/Lang/Holler/Hawkey/Rogler/ Biedermann/ASTIC trial group 


\section{Disclosure Statement}

None declared.

\section{Source of Funding}

None declared.

\section{Authorship Statement}

T.G. was responsible for acquisition and analysis of data, drafting of manuscript; S.L. and C.J.H. were responsible for acquisition of data and revision of manuscript. E.H. critically revised the manuscript. G.R. was responsible for study design, acquisition of data, and revision of manuscript. L.B. was responsible for study design, acquisition and analysis of data, and drafting of manuscript.

\section{References}

1 Narushima Y, Unno M, Nakagawara K, Mori M, Miyashita H, Suzuki Y, et al: Structure, chromosomal localization and expression of mouse genes encoding type III Reg, RegIII alpha, RegIII beta, RegIII gamma. Gene 1997; 185:159-168.

-2 Lasserre C, Christa L, Simon MT, Vernier P, Bréchot C: A novel gene (HIP) activated in human primary liver cancer. Cancer Res 1992;52:5089-5095.

- 3 Orelle B, Keim V, Masciotra L, Dagorn JC, Iovanna JL: Human pancreatitis-associated protein. Messenger RNA cloning and expression in pancreatic diseases. J Clin Invest 1992; 90:2284-2291.

-4 Gironella M, Iovanna JL, Sans M, Gil F, Peñalva $M$, Closa $D$, et al: Anti-inflammatory effects of pancreatitis associated protein in inflammatory bowel disease. Gut 2005;54 1244-1253.

5 Cash HL, Whitham CV, Behrendt CL, Hooper LV: Symbiotic bacteria direct expression of an intestinal bactericidal lectin. Science 2006; 313:1126-1130.

6 Zheng Y, Valdez PA, Danilenko DM, Hu Y, Sa SM, Gong Q, et al: Interleukin-22 mediates early host defense against attaching and effacing bacterial pathogens. Nat Med 2008;14 282-289.

7 Sanos SL, Bui VL, Mortha A, Oberle K, Heners C, Johner C, et al: RORgammat and commensal microflora are required for the differentiation of mucosal interleukin 22-producing NKp46+ cells. Nat Immunol 2009;10: 83-91.

-8 Wolk K, Kunz S, Witte E, Friedrich M, Asadullah K, Sabat R: IL-22 increases the innate immunity of tissues. Immunity 2004;21:241-254

-9 Ferrara JL, Levine JE, Reddy P, Holler E: Graft-versus-host disease. Lancet 2009;373: $1550-1561$.

10 Ferrara JL, Harris AC, Greenson JK, Braun TM, Holler E, Teshima T, et al: Regenerating islet-derived 3-alpha is a biomarker of gastro- intestinal graft-versus-host disease. Blood $>18$ Farge D, Labopin M, Tyndall A, Fassas A 2011;118:6702-6708

-11 Holler E, Rogler G, Herfarth H, Brenmoehl J, Wild PJ, Hahn J, et al: Both donor and recipient NOD2/CARD15 mutations associate with transplant-related mortality and GvHD following allogeneic stem cell transplantation. Blood 2004;104:889-894.

12 Holler E, Rogler G, Brenmoehl J, Hahn J, Herfarth $\mathrm{H}$, Greinix $\mathrm{H}$, et al: Prognostic significance of NOD2/CARD15 variants in HLA identical sibling hematopoietic stem cell transplantation: effect on long-term outcome is confirmed in 2 independent cohorts and may be modulated by the type of gastrointestinal decontamination. Blood 2006;107:4189-4193.

13 Vermeire S, Van Assche G, Rutgeerts P: Laboratory markers in IBD: useful, magic, or unnecessary toys? Gut 2006;55:426-431.

14 Mosli MH, Zou G, Garg SK, Feagan SG, MacDonald JK, Chande N, et al: C-reactive protein, fecal calprotectin, and stool lactoferrin for detection of endoscopic activity in symptomatic inflammatory bowel disease patients: a systematic review and meta-analysis. Am J Gastroenterol 2015;110:802-819.

15 Henderson P, Kennedy NA, Van Limbergen JE, Cameron FL, Satsangi J, Russell RK, et al: Serum C-reactive protein and CRP genotype in pediatric inflammatory bowel disease: in fluence on phenotype, natural history, and response to therapy. Inflamm Bowel Dis 2015; 21:596-605.

16 Pihusch M, Pihusch R, Fraunberger P, Pihusch V, Andreesen R, Kolb HJ, et al: Evaluation of C-reactive protein, interleukin-6, and procalcitonin levels in allogeneic hematopoietic stem cell recipients. Eur J Haematol 2006; 76:93-101.

17 Peyrin-Biroulet L, Reinisch W, Colombel JF, Mantzaris GJ, Kornbluth A, Diamond R, et al: Clinical disease activity, C-reactive protein normalisation and mucosal healing in Crohn's disease in the SONIC trial. Gut 2014;63:88-95.
Mancardi GL, Van Laar J, et al: Autologous hematopoietic stem cell transplantation for autoimmune diseases: an observational study on 12 years' experience from the European group for blood and marrow transplantation working party on autoimmune diseases. Haematologica 2010;95:284-292.

19 Hawkey CJ: Haemopoetic stem cell transplantation for severe resistant Crohn's disease: preliminary evidence for durable benefit. Gut 2014;63(suppl 1):A4

20 Hawkey CJ: 1162 controlled trial of immunoablation and autologous haemopoetic stem cell transplantation in Crohn's disease: interim report on behalf of the ASTIC trialists. Gastroenterology 2012;142:S-212.

21 Hawkey CJ, et al: Clinical and endocopic improvement following hemopoietic stem cell transplantation vs mobilisation alone in Crohn's disease. Gastroenterology 2013; 144(5 suppl 1):S-21-S-22.

22 Paczesny S, Braun TM, Levine JE, Hogan J, Crawford J, Coffing B, et al: Elafin is a biomarker of graft-versus-host disease of the skin. Sci Transl Med 2010;2:13ra2.

23 Singleton JW: The Crohn's disease activity index is a useful tool in clinical practice of gastroenterology. Inflamm Bowel Dis 1999;5: 138; discussion 141-143.

24 Harvey RF, Bradshaw JM: A simple index of Crohn's-disease activity. Lancet 1980;1:514.

25 Mary JY, Modigliani R: Development and validation of an endoscopic index of the severity for Crohn's disease: a prospective multicentre study. Groupe d'etudes thérapeutiques des affections inflammatoires du tube digestif (GETAID). Gut 1989;30:983-989.

-26 Takashima S, Kadowaki M, Aoyama K, Koyama M, Oshima $\mathrm{T}$, Tomizuka $\mathrm{K}$, et al: The Wnt agonist R-spondin1 regulates systemic graft-versus-host disease by protecting intestinal stem cells. J Exp Med 2011;208: 285-294. 